\title{
THE DIRECTIONAL DEPENDENCE OF COMETARY MAGNETIC ENERGY DENSITY IN THE QUASI-PARALLEL AND QUASI- PERPENDICULAR REGIMES
}

\author{
R. H. Miller,* T. I. Gombosi,* S. P. Gary** and D. Winske*** \\ * Space Physics Research Laboratory, Department of Atmospheric, Oceanic \\ and Space Sciences, The University of Michigan, Ann Arbor, Michigan 48109. \\ 214.3, U.S.A. \\ ** Space and Science Technology Division; *** Applied Theoretical Physics \\ Division, Los Alamos National Laboratory. Los Alamos, New Mexico 87545 \\ U.S.A.
}

\section{ABSTRACT}

The direction of propagation of low frequency magnetic fluctuations generated by cometary ion pick-up is examined by means of one-dimensional electromagnetic hybrid simulations. The newborn ions are injected at a constant rate, and the helicity and direction of propagation of magnetic fluctuations are explored for cometary ion injection angles of $0^{\circ}$ and $90^{\circ}$ relative to the solar wind magnetic field. The parameter $\eta$ represents the relative contribution of wave energy propagating in the direction away from the comet, parallel to the beam. For small (quasi-parallel) injection angles $\eta$ was to found to be of order unity, while for larger (quasi-perpendicular) angles $\eta$ was found to be of order 0.5 .

\section{INTRODUCTION}

The solar wind - comet interaction is very different from the solar wind interaction with other solar system bodies with gravitationally bound dense atmospheres and/or significant intrinsic magnetic fields. Cometary neutral atoms and molecules escape from the surface of the comet and move with velocities ranging from a few hundred meters per second to a few tens of kilometers per second, relative to the comet. As the neutrals migrate outwards, they become ionized through photoionization, electron impact or charge exchange with a characteristic ionization lifetime of $10^{5}$ $10^{7}$ seconds $/ 8 /$, thereby representing a large, "soft" obstacle for the supersonic and superalfvenic solar wind flow /1/.

The introduction of these newborn cometary ions represents an impulsive source of free energy in the solar wind. The initial velocity distribution is a strong function of the angle, $\alpha$, between the ambient magnetic field and the solar wind. When $\alpha \sim 0^{\circ}$ or very small, the velocity distribution is beam like, which can excite a variety of plasma instabilities $/ 5,13,14 /$ whose ensuing fluctuations contribute to pick-up by scattering and heating the newborn ions.

For $\alpha \sim 90^{\circ}$, the velocity distribution forms a ring distribution where the pick-up process is moderated by the motional electric field $/ 13,14,12$ / but other plasma instabilities also occur.

The predominant electromagnetic instability propagating parallel to the magnetic field in the quasi-parallel regime $\left(0^{\circ} \leq \alpha \leq 60^{\circ}\right)$ is the electromagnetic :on/ion right-hand resonant instability $/ 3,19,5,4 /$. This mode approximately satisfies the cyclotron resonance condition,

$$
\omega_{\mathrm{r}}-\mathbf{k} \cdot \mathbf{v}_{\mathrm{ob}}+\Omega_{\mathrm{b}}=0
$$

where the wave frequency in the center of mass frame is $\omega_{t}, \mathbf{k}$ is the wave vector, $\mathbf{v}_{\mathrm{ob}}$ is the initial cometary ion velocity in the same frame and $\Omega_{b}$ is the cyclotron frequency of the beam component. In the quasi-perpendicular regime $\left(\alpha>60^{\circ}\right)$, the left-hand anisotropy ion cyclotron mode is dominant.

In this paper, we consider the directional dependence of the fluctuating magnetic field energy density generated by cometary ion pick-up. The parameter $\eta$ determines the fraction of wave energy that propagates parallel to the beam, away from the comet. $\eta$ is investigated for two representative cases: (1) a pure oxygen beam velocity distribution, $\alpha=0^{\circ} ;(2)$ a ring distribution at $\alpha=90^{\circ}$. $\eta$ is characterized as a function of injection angle which will help elucidate the transition from the quasi-parallel to the quasi-perpendicular regime. The parameter $\eta$ also helps link hybrid simulations where the pick-up ion instabilities are modeled to test-particle calculations which model particle acceleration via quasilinear theory $/ 2 \%$.

We use a one-dimensional hybrid simulation similar to that previously employed by Gary et al. $/ 5,6 /$ to simulate the cometary ion/solar wind interaction. The cometary ions are continuously injected as in the Gary et al. /5,6/ simulations. The new element in this present work is that we have included a diagnostic which divides the total wave spectrum into two components: (1) the energy propagating antiparallel to the beam, i.e., towards the comet and away from the sun, $P_{+}$, in the solar wind frame; (2) the energy propagating parallel to the beam, i.e., away from the comet towards the sun, $\mathrm{P}_{-}$, in the solar wind frame, and we have separated the fluctuating magnetic field into Fourier modes and (positive/negative) helicity components /15/ which enable the determination of directional properties, wave 
properties, and mode types. The parameter $\eta$ is defined as the fraction of energy in waves propagating in the forward direction

$$
\eta=\frac{P-(k)}{P_{\text {tot }}(k)}
$$

where the total energy is $P_{t o t}(k)=P_{+}(k)+P_{-}(k)$.

In Section 2 of this paper, we describe the simulations and a brief discussion of the dominant instabilities for $\mathbf{k} \times \mathbf{B}_{\mathrm{o}}=0$. In Section 3, the results of the simulations are examined. Section 4 contains a discussion of these numerical results.

\section{SIMULATION}

The hybrid simulations used in this paper and in earlier simulations of Gary et al. /5,6/ and Winske et al. /18/ treats the electrons as a massless, charge neutralizing fluid and the ions as discrete particles /17/. Since the electrons are massless, quasi-neutrality is maintained and the low frequency approximation (Darwin) is adopted. The interaction between the ions and the electric and magnetic fields are calculated self-consistently. The simulation is onedimensional in space, but the particle velocities and electromagnetic fields are fully three-dimensional. Periodic boundary conditions for both particles and fields are used, consistent with the idea of the simulation being done over a limited region of space in which the injection rate is constant.

Our notation and definitions are consistent, for the most part, with those of Gary et al. 15,6/. The injected oxygen ions are distributed randomly through one-dimensional space with constant injection rate and injection velocity, $\mathbf{v}_{\mathrm{ob}}=\mathbf{x v _ { \mathrm { ob } }}$, thus simulating the ionization of cometary neutrals, and the subsequent appearance of new cold ions in the solar wind. We only consider the case when $\mathbf{k} \times \mathbf{B}_{\mathrm{o}}=0$, because the instabilities are a maximum at this angle of propagation $13 /$. Thus, our simulations do not describe physical effects due to oblique propagation and associated compressibility of the fluctuations.

Our simulations involves three species: (1) solar wind protons, (2) solar wind electrons, and (3) a tenuous injected oxygen component. The simulations have a cold oxygen ion beam, $\beta_{0+}=0$, with a Maxwellian solar wind plasma, characterized by $\beta_{c}=1.0$ for the electrons and protons. The injected ions have an injection velocity which is $v_{0 b}=10 \mathrm{v}_{A}$ where $\mathrm{v}_{\mathrm{A}}$ is the Alfven velocity and where we have taken $\mathrm{v}_{\mathrm{A}} / \mathrm{c}=10^{-4}$. The injection angle and timestep have been taken as, $\alpha=0^{\circ}$ or $90^{\circ}$, and $\Omega_{\mathrm{p}} \Delta \mathrm{t}=0.4$, respectively. The simulation box size is chosen to be three times longer than the wavelength at the maximum linear growth rate of the ion/ion right-hand polarized resonant instability, $L=2400 \mathrm{c} / \omega_{\mathrm{p}}$ and the number of cells is NZ=128. From this, the dominant Fourier mode of the fluctuating magnetic field energy density, at $\alpha=0^{\circ}$, should be the $N=3$ mode. For $\alpha=90^{\circ}$, the ion/ion right-hand instability is not triggered but the lefthand ion cyclotron anisotropic mode is instead excited. In quasi-perpendicular regimes $\left(70^{\circ} \leq \alpha \leq 90^{\circ}\right)$, cometary ion instabilities no longer satisfy Eq. (1) and mode identification is more difficult, although Glassmeier et al. $7 /$ repon some evidence of left-hand polarization, commensurate with the prediction of Gary et al. $15,6 /$.

\section{RESULTS}

This section presents the simulation results, in particular characterizing the propagation parameter $\eta$ as a function of injection angle $\alpha$. All simulations reported in this paper use the parameters stated in the caption of Figure 1; only $\alpha$ is varied. Figure la shows $\eta$ as a function of time for the $\alpha=0^{\circ}$ case for the first 6 Fourier modes in the system. At early times, the spectrum (not shown) is peaked about mode $N=4$ but later in time, by $150 \Omega_{\mathrm{p}}{ }^{-1}$, the dominant mode shifts to the ion/proton right-hand wavevector, $\mathrm{N}=3$, and remains the dominant mode throughout the simulation. The parameter $\eta$ for $\mathrm{N}=3$ evolves from the background value of 0.5 to 0.98 . With $\eta$ on the order of unity, one would expect to see most of the wave energy propagating parallel to the beam and away from the comet.

In Figure $1 \mathrm{~b}$, the positive and negative helicity components of the $\mathrm{N}=3$ Fourier mode of the magnetic field are shown as a function of distance along the magnetic field. The fluctuating magnetic field spectra have been stacked on top of each other allowing for the observation of magnetic waves propagating in the system. The panel on the right of Figure $1 \mathrm{~b}$ contains the positive helicity waves where the negative helicity waves are in the left panel. The ion/proton right-hand resonant instability has positive helicity and right-hand polarization and can thus be identified as the mode in the right panel. The initial "bumps" in the magnetic field corresponds to the exponential regime where the phase velocity is apparently small. The magnetic energy density not presented here undergoes exponential temporal growth initially and then linear temporal growth ensues $/ 5,6 /$. As one moves through the exponential oscillations which are due to magnetic particle trapping, the ion/proton right-hand resonant mode relaxes to the appropriate phase velocity which is approximately the Alfven speed.

In the left panel of Figure $1 \mathrm{~b}$, the nonresonant instability can be seen with a significantly reduced amplitude much later in time. The nonresonant instability has negative helicity and propagates antiparallel to the beam in the center of mass frame. The frequency of this mode has been Doppler shifted to positive values in the solar wind rest frame and hence propagates parallel to the beam in Figure 1b, i.e., the nonresonant mode has changed polarization: right- to left-hand.

As the injection angle is increased from $0^{\circ}$ to $90^{\circ}$, the physics moves from the quasi-parallel to quasi-perpendicular regime and the nature of the dominant instability changes, since linear theory predicts that at injection angles greater than $70^{\circ}$, left-hand polarized waves become dominant $/ 16 \%$. The values of $\eta$ for the first 6 Fourier modes are found in Figure 2a. All 6 modes have $\eta$ on the order of 0.5 for the $90^{\circ}$ case indicating that the energy is propagating equally parallel and antiparallel to the beam. The shorter wavelengths, not depicted here, have $\eta$ 's that are of order unity but 

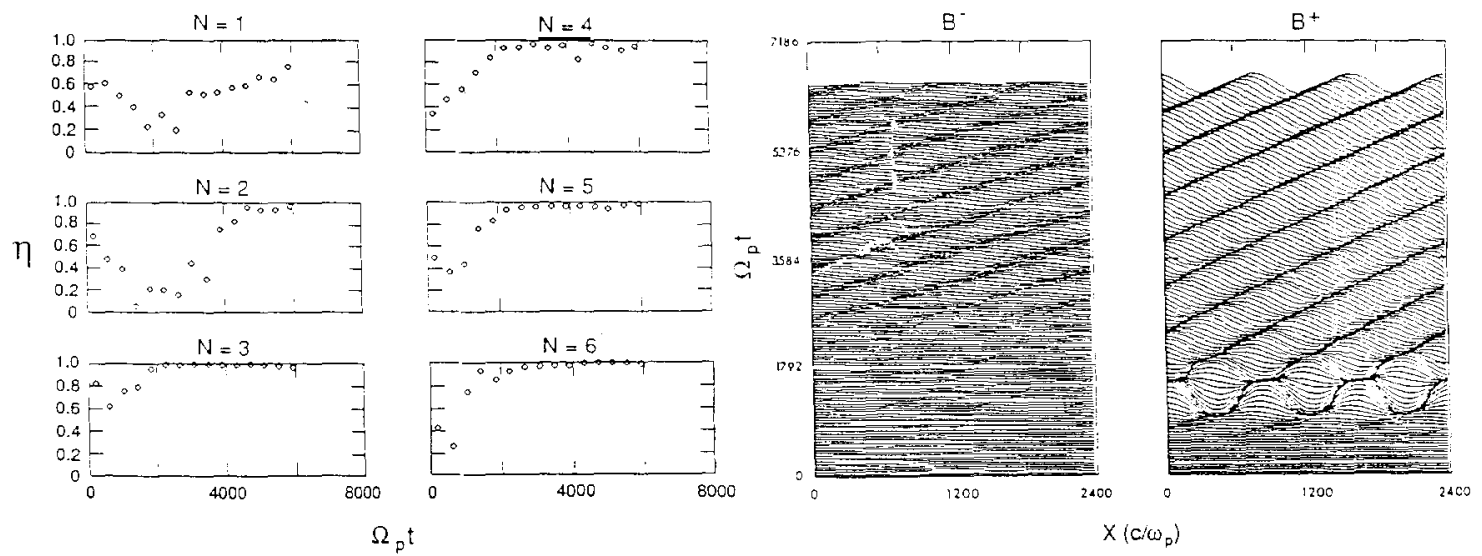

Figure 1: In Figure 1a, the $\eta$ 's for the first six Fourier modes are displayed as a function time for $\alpha=0^{\circ}$. The initial parameters used in this simulation and all other simulations are $m_{b}=16 \mathrm{~m}_{c}, v_{o b}=10 \mathrm{v}_{\mathrm{A}}, \Lambda_{b}=5.0 \times 10^{-7} \Omega_{\mathrm{p}}, \mathrm{T}_{\mathrm{e}}=\mathrm{T}_{\mathrm{c}}$, $\beta_{\mathrm{c}}=1.0, \mathrm{v}_{\mathrm{O}}=0, \mathrm{v}_{\mathrm{A}} / \mathrm{c}=10^{-4}$ with $\Omega_{\mathrm{p}} \Delta \mathrm{t}=0.4, \mathrm{NZ}=128$, and $\mathrm{L} \omega_{\mathrm{d}} / \mathrm{c}=2400$ : $\mathrm{b}$ denotes the oxygen beam population and $\mathrm{c}$ represents the proton core constituert and $\mathrm{e}$ is for the electrons. Figure $1 \mathrm{~b}$ contains the fluctuating magnetic field for $\alpha=0^{\circ}$, at sequential times, which are stacked on top of each other with the vertical axis corresponds to time and the horizontal axis corresponds to distance along the background magnetic field. The magnetic field has been filtered to display mode $\mathrm{N}=3$ which corresponding to the wavelength of the ion/proton right-hand resonant instability. The right and left panel contains the positive and negative helicities, respectively.
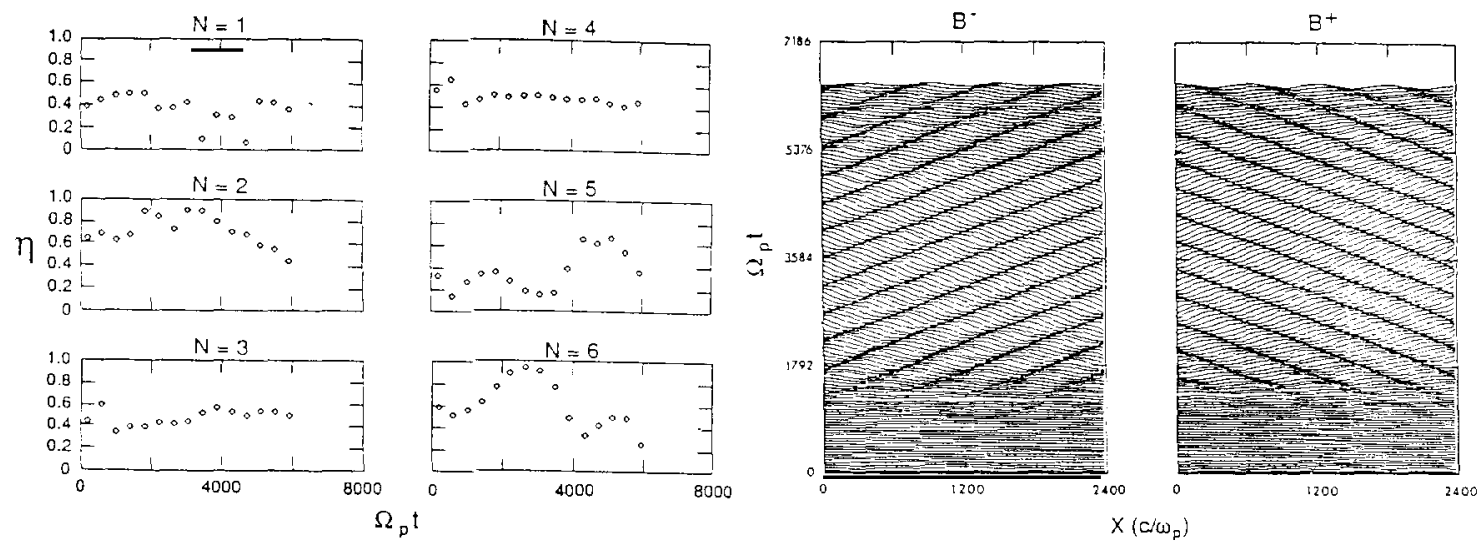

Figure2: In Figure 2a, the $\eta$ 's for the first six Fourier modes are shown for an injection angle of $90^{\circ}$. Figure $2 b$ shows the fluctuating magnetic field for $\alpha=90^{\circ}$ which is displayed for mode $N=4$, where the positive and negative helicities are found in the right and left panels, respectively.

do not pitch angle scatter the ions effectively. The ion cyclotron ring instability for a zero parallel beam temperature has its largest growth rate for short wavelengths /4/. Initially, the shorter wavelengths are excited but as the parallel beam temperature increases the ins:ability evolves to longer wavelength. We have concentrated on the longer wavelength modes that are responsible for effective pitch-angle scattering.

Examining mode 4, Figure $2 \mathrm{~b}$, one finds that there is an equal amount of energy propagating in the parallel and antiparallel direction. This is consistent with the injection of free-energy perpendicular to the direction of propagation of these waves. The velocity distribution of cometary ions is symmetric for $\alpha \approx 90^{\circ}$ producing equal growth rates for the two left-hand polarized ion-cyclotron anisotropic modes with positive and negative helicities. This is supported by Figure $2 \mathrm{a}$ where the $\mathrm{N}=4$ mode has $\eta$ on the order of 0.5 .

\section{SUMMARY}

In this paper, we have examined the clirection of propagation of the field aligned electromagnetic waves generated in the interaction of newborn cometary ions with the solar wind. Numerical simulation techniques have been used to investigate $\eta$, the ratio of wave energy propagating sunward in the solar wind frame to the total wave energy, when the cometary ions are injected at a constant rate to model the interaction process of a solar wind parcel traveling through the cometary environment. Two limiting cases have been examined, characterized by the angle of injection, $\alpha$, of the newborn ions with respect to the magnetic field. For $\alpha=0^{\circ}$, corresponding to the case where the cometary ions form a beam-like distribution, we find $\eta \approx 0.98$. Consistent with linear theory, the dominant wave mode in this "quasi-parallel regime" is readily identified as the right-hand polarized resonant ion/ion instability. Because the instability arises from a cyclotron resonant interaction, the waves are primarily generated in the direction of the beam. 
In the opposite limit, $\alpha=90^{\circ}$ (quasi-perpendicular regime), $\eta$ for the dominant mode is approximately 0.5 . In this case, the instability is a left-hand polarized ion cyclotron mode. It is driven by a ring-like nature of the cometary ion velocity distribution and according to linear theory, propagates equally along and against the magnetic field direction, consistent with the measured value of $\approx 0.5$. Analysis of a number of other cases shows that in the quasi-parallel regime $\left(0^{\circ} \leq \alpha \leq 60^{\circ}\right) \eta$ for the dominant mode is typically 0.98 , where for the quasi-perpendicular regime $\left(70^{\circ} \leq \alpha \leq 90^{\circ}\right), \eta$ is about 0.5 . These results are consistent with the limiting case described in this paper. Moreover, such results are significantly for estimating the effectiveness of the second order Fermi acceleration process in energizing the cometary ions.

Acceleration theories $19,10,11 /$ assume parallel propagating electromagnetic waves, which are the long-wavelength limit of the cyclotron resonant modes we have shown dominate in the quasi-parallel regime, and take $\eta=0.80$. Using a more realistic value of $\eta \approx 0.98$ would decrease the relative population of the two assumed sets of wave scattering centers and hence reduce the effectiveness of the acceleration process. In the limit of quasi-perpendicular injection, where the value of $\eta$ observed in the simulation is much smaller, the effectiveness of the acceleration would be increased, although again the maximum wave power resides at wavelengths shorter than those assumed in the theory.

Acknowledgments: This work was supported at the University of Michigan by NASA grants NAGW-2162 and NAGW-1366. Research at Los Alamos was done under the auspices of the U.S. Department of Energy and was supported in part by the NASA Research and Analysis Program at the Laboratory and by the CRRES program under NASA.

\section{REFERENCES}

1. Biermann, L., B. Brosowski, and H.U. Schmidt, The interaction of solar wind with a comet, Sol. Phys., 1, 254, 1967.

2. Cravens, T.E., Test particle calculations of the pick-up ions in the vicinity of comet Giacobini-Zinner, Planet. Space Sci., 37, 1169, 1989

3. Gary, S.P., C.W. Smith, M.A. Lee, M.L. Goldstein, and D.W. Forslund, Electromagnetic ion beam instabilities, Phys. Fluids, 27, 1852, 1984.

4. Gary, S.P., C.D. Madland, Electromagnetic ion instability in a cometary environment, J. Geophys. Res., 93, 235,1988

5. Gary, S.P., C.D. Madland, N. Omidi, and D. Winske, Computer simulations of two-pickup-ion instabilities in a cometary environment, J. Geophys. Res., 93, 9584, 1988.

6. Gary, S.P., K. Akimoto, and D. Winske, Computer simulations of cometary-ion/ion instabilities and wave growth, J. Geophys. Res., 94, 3513, 1989.

7. Glassmeier, K.-H., A.J. Coates, M.H. Acuna, M.L. Goldstein, A.D. Jonhstone, F.M. Neubauer, and H. Reme, Spectral characteristics of low-frequency plasma turbulence upstream of comet P/Halley, J. Geophys. Res., 94, 37, 1989

8. Gombosi, T.I., A.F. Nagy, and T.E. Cravens, Dust and neutral gas modeling of the inner atmospheres of comets, Rev. Geophys, $24,667,1986$.

9. Gombosi, T.I., Preshock region acceleration of implanted cometary $\mathrm{H}^{+}$and $\mathrm{O}^{+}, J$. Geophys. Res., 93, 35, 1988.

10. Gombosi, T.I., K. Lorencz, and J.R. Jokipii, Combined first- and second-order Fermi acceleration in cometary environments, J. Geophys. Res., 94, 15011, 1989.

11. Isenberg, P.A., Energy diffusion of pickup ions upstream of comets, J. Geophys. Res., 92, 8795, 1987.

12. Kimmel, C.D., J.G. Luhmann, J.L. Phillips, and I.A. Fedder, Characteristics of cometary pick-up ions in a global model of Giacobini-Zinner, J. Geophys. Res., 92, 8536, 1987.

13. Omidi, N., and D. Winske, Simulation of the solar wind interaction with the outer regions of the coma, Geophys. Res. Lett., 13, 397, 1986.

14. Omidi, N., and D. Winske, A kinetic study of the solar wind mass loading and cometary bow shocks, $J$. Geophys. Res., 92, 13409, 1987.

15. Terasawa, T., M. Hoshino, J.-I. Sakai, and T. Hada, Decay instability of finite-amplitude circularly polarized alfven waves: A numerical simulation of stimulated brillouin scattering, J. Geophys. Res., 91, 4171, 1986.

16. Thorne, R.M., and B.T. Tsurutani, Resonant interaction between cometary ions and low frequency electromagnetic waves, Planet. Space Sci., 35, 1501, 1987.

17. Winske, D., and M.M. Leroy, Diffuse ions produced by electromagnetic ion beam instabilities, J. Geophys. Res., 89. 2673, 1984

18. Winske, D., C.S. Wu, Y.Y. Li, and G.C. Zhou, Collective capture of released lithium ions in the solar wind, $J$. Geophys. Res., 89, 7327, 1984.

19. Winske, D., and S.P. Gary, Electromagnetic instabilities driven by cool heavy ion beams, J. Geophys. Res., 91, $6825,1986$. 